\title{
Locomotion coincides with c-Fos expression in related areas of inferior olive and cerebellar nuclei in the rat
}

\author{
Tom J.H. Ruigrok*, Hans van der Burg, Erika Sabel-Goedknegt \\ Department of Anatomy, Erasmus University Rotterdam, P.O. Box 1738, 3000 DR Rotterdam, The Netherlands
}

Received 19 June 1996; revised version received 3 July 1996; accepted 10 July 1996

\begin{abstract}
Rats that had been walking on a rotating drum for a period of $75 \mathrm{~min}$, demonstrated expression of the immediate early gene c-Fos in specific areas of the inferior olive and cerebellar nuclei. Non-walking control rats did not show consistent Fos-like immunoreactive labelling in these nuclei. Fos-like immunoreactive olivary neurons were consistently found in the caudolateral parts of the dorsal accessory olive and in its dorsal fold, and within specific areas of the medial accessory and principal olives. In the cerebellar nuclei, immunoreactive cells were found in the medial part of the anterior interposed nucleus, in the interstitial cell groups, and within specific parts of the medial, posterior interposed, and lateral nuclei. The lateral vestibular nucleus as well as the ventral part of the magnocellular red nucleus also displayed positive neurons. Most of these positive areas are known (1) to be related to the spinal cord and (2) to be anatomically linked. These results are in line with a presumptive role of the olivo-cerebellar system in ongoing movement control.
\end{abstract}

Keywords: Immediate early gene; Cerebellum; Walking; Motor control; Climbing fibre collaterals; Lateral vestibular nucleus; Red nucleus

The monitoring of the expression of immediate early genes such as c-Fos has proven to be a useful tool to investigate functional circuits in the brain and spinal cord. Jasmin et al. [12] recently showed that a prolonged period of walking resulted in an enhanced expression of Fos-like immunoreactivity in the rat spinal cord, dorsal column nuclei and cerebellum. Within the cerebellum a profound increase in Fos expression was apparent within the granule cell layer and in parts of the molecular layer. The authors conclude that this enhanced c-Fos expression corresponded to an increased activity in several direct and indirect spinocerebellar pathways which are known to terminate as mossy fibres in the granule cell layer. In the present study, we used a similar approach to investigate c-Fos expression in the inferior olive (IO) which, being the sole source of climbing fibres, represents another important afferent system of the cerebellum. Moreover, since the cerebellum may use its sensory input to exert some kind of control on motor performance [4], the locomotion-related

\footnotetext{
* Corresponding author. Tel.: +31 10 4087296; fax: +31 104365780 ; email: ruigrok@anat.fgg.eur.nl
}

expression of c-Fos was monitored in the cerebellar nuclei (CN), which constitute the main output system of the cerebellum.

A total of ten male Wistar rats, weighing between 250 and $300 \mathrm{~g}$ were divided into five pairs. Each pair was trained to walk on a rotating drum (custom-build, diameter $30 \mathrm{~cm}$ ), which could be activated by a foot switch (speed $10 \mathrm{~m} / \mathrm{min}$ ). During each training session the rat was kept for $1 \mathrm{~h}$ on the drum with an alternating $1 \mathrm{~min}$ on, $4 \mathrm{~min}$ off training schedule. After six daily training sessions, all rats had learned to walk on the drum, maintaining a more or less continuous and even pace every time it was activated. The following day, one rat of each pair (the experimental animal) had to perform on the rotating drum for $75 \mathrm{~min}$ with a $14 \mathrm{~min}$ on, $1 \mathrm{~min}$ off activation schedule. The other rat stayed on the inactivated drum for $75 \mathrm{~min}$, and served as a control.

Immediately after the test period the rats were given an overdose of sodium pentobarbital $(120 \mathrm{mg} / \mathrm{kg}$ i.p.) and were transcardially perfused with physiological saline $(400 \mathrm{ml})$, followed by a phosphate-buffered paraformaldehyde $(4 \%)$ solution $(1000 \mathrm{ml})$. The brain and spinal cord 
were extracted, post-fixed for $2-6 \mathrm{~h}$, embedded in gelatin and immersed in $30 \%$ sucrose in phosphate buffer. Transverse sections $(40 \mu \mathrm{m})$ were cut on a freezing microtome and incubated in sheep anti-c-Fos (Cambridge Research Biochemicals; dilution 1:2000 in phosphate-buffered saline containing $0.4 \%$ Triton X-100) for $48 \mathrm{~h}(4 / \mathrm{C})$. Sections of the experimental and control animal were always incubated simultaneously in separate vials. Following subsequent incubation in biotinylated donkey anti-sheep (Jackson) for $2 \mathrm{~h}$ and ABC Elite (Vector) for $2 \mathrm{~h}$, the sections were processed for standard peroxidase-DAB histochemistry [10]. Finally, the sections were mounted, lightly counterstained with thionine and analysed with an Olympus microscope equipped with a Lucivid miniature monitor. Neurons with Fos-like immunoreactive (FLI) nuclei in $\mathrm{IO}$ and $\mathrm{CN}$ were identified using a $20 \times$ objective and were plotted with Neurolucida software (MicroBrightField, Inc.).

The experimental and control rat of each pair displayed marked and consistent differences with respect to the number and distribution of FLI neurons. The control animals displayed relatively few positive neurons in spinal cord, brainstem and cerebellum, whereas the period of walking corresponded with the induction of large numbers of positive FLI neurons throughout the spinal cord and in several brainstem areas such as the dorsal column nuclei, reticular formation, lateral reticular nucleus, trigeminal nucleus, and within the granule and molecular layers of the cerebellum (cf. [12]).

In all five experimental animals, and contrary to their controls, FLI neurons were also systematically encountered within the confines of the IO as well as of the $\mathrm{CN}$ (Fig. 1). The location of FLI neurons was essentially bilaterally symmetric but differed somewhat between cases with respect to the total number of positive neurons, the size of the nuclear region displaying the positive neurons, and the intensity of the FLI labelling. However, in all experimental cases the identity of the positive regions was similar. Case 645 is described as an example (Fig. 2 ). Within the IO, positive neurons were predominantly found in the dorsal fold of the dorsal accessory olive (DAO), within the lateral and central parts of the DAO, in the central and caudal parts of the medial accessory olive (MAO), in the caudal part of the subnucleus beta, and, more sparsely, within the lateral bend of the principal olive (PO). In the CN, FLI neurons were observed in the rostromedial part of the anterior interposed nucleus (AIN), within the area interspersed between the medial and interposed nuclei (the interstitial cell groups (ICG) [6]), in the medial part of the posterior interposed nucleus (PIN), and within the caudomedial part of the medial cerebellar nucleus (MCN). More widespread and sparse concentra-
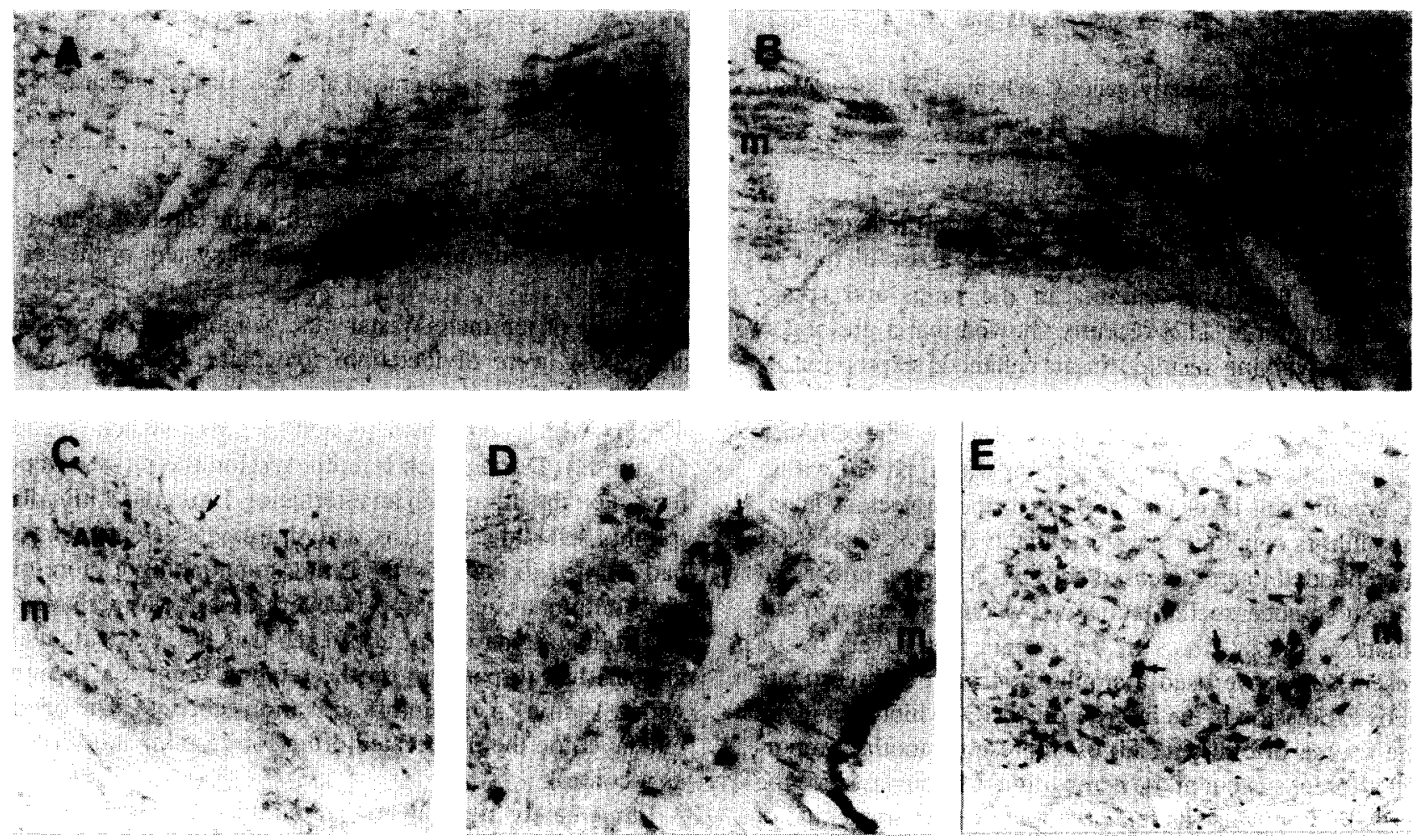

Fig. 1. Photomicrographs of c-Fos immunostained and thionine-counterstained sections of case 650 (experimental rat: A,C,D,E) and of case 651 (control rat: B). Typical FLI neurons are indicated with arrows. (A) c-Fos positive neurons in the dorsal fold of the DAO and in the MAO; (B) lack of c-Fos labelling in a comparable region of the inferior olivary complex in a control case; (C) FLI neurons in the AIN; (D) FLI neurons in the LVN; (E) FLI neurons in the ventromedial part of the red nucleus. Bar in (A) equals $100 \mu \mathrm{m}$ for (A,B) and $200 \mu \mathrm{m}$ for (C,D,E). M, medial. 
Inferior olive

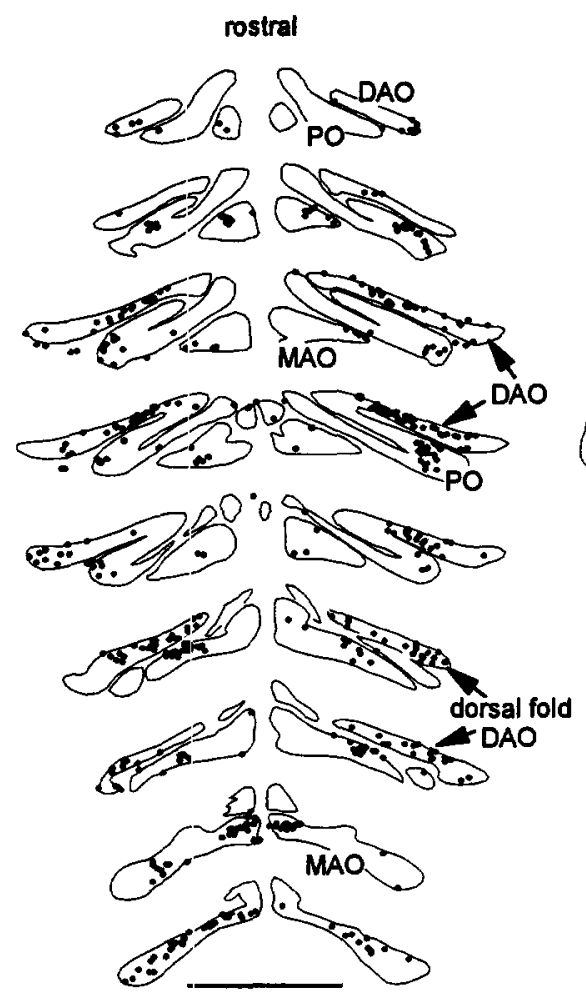

Cerebellar nuclei

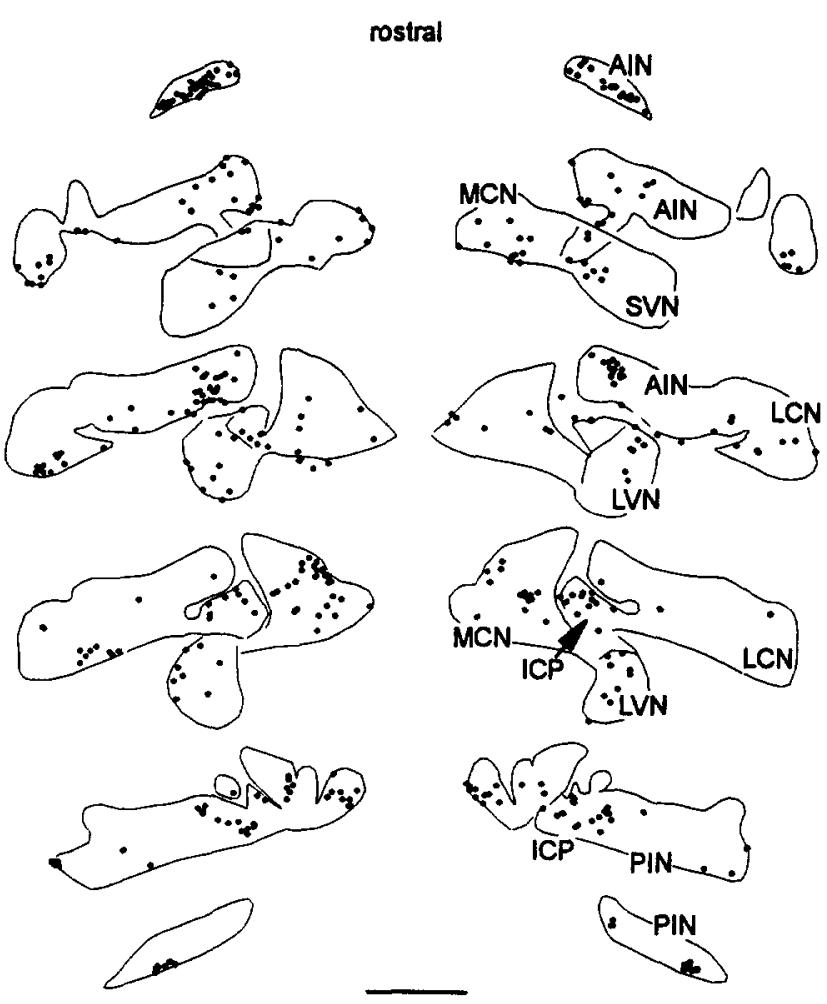

Fig. 2. Diagrams indicating the location of c-Fos immunoreactive neurons in the inferior olive and cerebellar nuclei of case 645 (experimental rat). Drawings are based on serial transverse sections taken at $320 \mu \mathrm{m}$ intervals. Each dot represents one c-Fos immunoreactive nucleus. Identification of subnuclei according to Ruigrok and Voogd [18]. Bar represents $1000 \mu \mathrm{m}$.

tions of positive nuclei were found throughout the $\mathrm{MCN}$ and within the ventral part of the lateral cerebellar nucleus (LCN). The lateral vestibular nucleus (LVN), which, like the $\mathrm{CN}$, receives a direct projection from the cerebellar cortex [22] also displayed many FLI profiles (see Fig. 1D).

These results indicate that a relatively long period of locomotion is related to the induction of c-Fos positive neurons within specific regions of the IO and $\mathrm{CN}$. Since the CN (and LVN) function as output stations of the cerebellum, it is notable that most of the regions displaying cFos positive neurons are at the origin of major projections to the spinal cord. The LVN is the origin of the lateral vestibulospinal spinal tract which descends throughout the whole spinal cord [15]. The AIN, via its strong projection to the magnocellular red nucleus, is capable of influencing activity in the rubrospinal tract [20]. Interestingly, FLI neurons within the red nucleus were specifically observed in its ventrocaudal part (Fig. 1E), which corresponds with the location of the FLI neurons in the medial part of the AIN, and which has been implicated in the control of the hindlimb [7,8]. The MCN, by way of its projections to vestibular nuclei and reticular formation, may also influence activity in vestibulospinal and reticulospinal pathways $[15,22]$. More recently, cervical projections originating from the ICG and medial PIN have been described $[3,11]$. A potential relation between the FLI expression in the ventral LCN and the spinal cord is more speculative, but could involve the superior colliculus and/or thalamus, sensorimotor cortex and pyramidal tract. In concurrence with the apparent relation between the FLI areas in the $\mathrm{CN}$ and the spinal cord, it may be noted that several of the FLI areas within the IO, such as the DAO, its dorsal fold, and the caudal MAO, are known to receive projections from the spinal cord $[5,14]$. The FLI neurons in nucleus beta most likely are related to vestibular functions [13] operating during locomotion.

Apart from the relation with the spinal cord, it is of special interest that the FLI regions in IO and $\mathrm{CN}$ are thought to be closely related. A reciprocal organization has been demonstrated between the nucleo-olivary projection [18] and the olivary innervation of the $\mathrm{CN}$ by climbing fibre collaterals $[16,21]$. Thus, the dorsal fold of the DAO is reciprocally connected to the LVN; the DAO to the AIN; the central part of the MAO to the ICG; the bend of the PO to the bend ( = ventral part) of the LCN; and the nucleus beta to specific areas in the MCN.

Despite the fact that the organization of the anatomical connections between the IO and $\mathrm{CN}$ is most likely at the basis of their specific expression, the exact mechanism of this conjunct FLI expression in IO and $\mathrm{CN}$ is more speculative. Is induction in one area dependent on increased activity in the other, or are both expressed as the result of 
enhanced activity in a, common, third system? It will be obvious that for the execution of complex behaviour such as locomotion many systems will interact with one another [12]. Nevertheless, it seems possible that the increased FLI expression in olivary neurons could be induced by way of spino-olivary pathways. An increased olivary activity could affect, by way of the excitatory olivary collaterals, related $\mathrm{CN}$ regions [1,21]. Increased activity of neurons supplying mossy fibre collaterals (e.g. the lateral reticular nucleus [17]) to the same $\mathrm{CN}$ regions could support their FLI response. In addition, increased activity of olivary neurons may result in depression of the simple spike activity of Purkinje cells which, in turn, may lead to disinhibition (i.e. in increased activity) of the related $\mathrm{CN}$ areas (see e.g. $[2,9,19])$.

[1] Audinat, E., Gähwiler, B.H. and Knöpfel, T., Excitatory synaptic potentials in neurons of the deep nuclei in olivo-cerebellar slice cultures, Neuroscience, 49 (1992) 903-911.

[2] Batini, C., Benedetti, F., Buisseret-Delmas, C., Montarolo, P.G. and Strata, P., Metabolic activity of intracerebellar nuclei in the rat: effects of inferior olive inactivation, Exp. Brain Res., 54 (1984) 259-265.

[3] Batini, C., Buisseret-Delmas, C., Compoint, C. and Volta-Pinardi, U., Vestibular, prepositus hypoglossi and cerebellar projections to the spinal cord, Eur. J. Neurosci., Suppl., 8 (1995) 97.

[4] Bloedel, J.R. and Bracha, V., On the cerebellum, cutaneomuscular reflexes, movement control and the elusive engrams of memory, Behav. Brain Res., 68 (1995) 1-44.

[5] Boesten, A.J.P. and Voogd, J., Projections of the dorsal column nuclei and the spinal cord on the inferior olive in the cat, J. Comp. Neurol., 161 (1975) 215-238.

[6] Buisseret-Delmas, C., Yatim, N., Buisseret, P. and Angaut, P., The $\mathrm{X}$ zone and $\mathrm{CX}$ subzone of the cerebellum in the rat, Neurosci. Res., 16 (1993) 195-207.

[7] Daniel, H., Angaut, P., Batini, C. and Billard, J.M., Topographic organization of the interpositorubral connections in the rat. A WGA-HRP study, Behav. Brain Res., 28 (1988) 69-70.

[8] Daniel, H., Billard, J.M., Angaut, P. and Batini, C., The interpositorubrospinal system. Anatomical tracing of a motor control pathway in the rat, Neurosci. Res., 5 (1987) 87-112.

[9] De'Sperati, C., Montarolo, P.G. and Strata, P., Effects of inferior olive inactivation and lesion on the activity of medial vestibular neurons in the rat, Neuroscience, 53 (1993) 139-147.

[10] Graham, R.C. and Karnovsky, M.J., The early stages of absorption of injected horseradish peroxidase in the proximal tubules of the mouse kidney. Ultrastructural cytochemistry by a new technique, $\mathrm{J}$. Histochem. Cytochem., 14 (1966) 291-302.

[11] Horn, K.M., Porter, C.M. and Gibson, A.R., Spinal connections of cat posterior interpositus, Soc. Neurosci. Abstr., 21 (1995) 1190.

[12] Jasmin, L., Gogas, K.R., Ahlgren, S.C., Levine, J.D. and Basbaum, A.I., Walking evokes a distinctive pattern of Fos-like immunoreactivity in the caudal brainstem and spinal cord of the rat, Neuroscience, 58 (1994) 275-286.

[13] Kaufman, G.D., Anderson, J.H. and Beitz, A., Activation of a specific vestibulo-olivary pathway by centripetal acceleration in rat, Brain Res., 562 (1991) 311-317.

[14] Matsushita, M., Yaginuma, H. and Tanami, T., Somatotopic termination of the spino-olivary fibers in the cat, studied with the wheat germ agglutinin-horseradish peroxidase technique, Exp. Brain Res., 89 (1992) 397-407.

[15] Rubertone, J.A., Mehler, W.R. and Voogd, J., The vestibular nuclear complex. In G. Paxinos (Ed.), The Vestibular Nuclear Complex, Academic Press, Sydney, 1995, pp. 773-796.

[16] Ruigrok, T.J.H., Cerebellar nuclei: the olivary connection. In C.I. De Zeeuw, P. Strata and J. Voogd (Eds.), The Cerebellum: From Structure to Control, Progress in Brain Research, 1997, in press.

[17] Ruigrok, T.J.H., Cella, F. and Voogd, J., Connections of the lateral reticular nucleus to the lateral vestibular nucleus in the rat. An anterograde tracing study with Phaseolus vulgaris leucoagglutinin, Eur. J. Neurosci., 7 (1995) 1410-1413.

[18] Ruigrok, T.J.H. and Voogd, J., Cerebellar nucleo-olivary projections in rat. An anterograde tracing study with Phaseolus vulgarisleucoagglutinin (PHA-L), J. Comp. Neurol., 298 (1990) 315-333.

[19] Strata, P., Inferior olive: functional aspects. In J.R. Bloedel, J. Dichans and W. Precht (Eds.), Inferior Olive: Functional Aspects, Springer-Verlag, Berlin, 1984, pp. 230-246.

[20] Tarnecki, R., Functional connections between neurons of interpositus nucleus of the cerebellum and the red nucleus, Behav. Brain Res., 28 (1988) 117-125.

[21] van der Want, J.J.L., Wiklund, L., Guegan, M., Ruigrok, T. and Voogd, J., Anterograde tracing of the rat olivocerebellar system with Phaseolus vulgaris-leucoagglutinin (PHA-L). Demonstration of climbing fiber collateral innervation of the cerebellar nuclei, J. Comp. Neurol., 288 (1989) 1-18.

[22] Voogd, J., The cerebellum. In G. Paxinos (Ed.), The Cerebellum, Academic Press, Sidney, 1995, pp. 309-350. 